\section{The effect of topical anti-muscarinic agents on subfoveal choroidal thickness in healthy adults}

V Öner, A Bulut and $\mathrm{K}$ Öter

\begin{abstract}
Purpose To investigate the effects of tropicamide and cyclopentolate, which are two anti-muscarinic agents commonly used in the ophthalmologic practice, on subfoveal choroidal choroidal thickness (ChT) in healthy adults.

Methods A total of 74 healthy adult subjects were enrolled in the study. Subjects were randomly divided into two groups: (1) cyclopentolate group $(n=37)$ in which the right eye (study eye) of each subject received topical cyclopentolate $1 \%$, and the fellow eye (control eye) received artificial tears and (2) tropicamide group $(n=37)$ in which the right eye (study eye) of each subject received topical tropicamide $1 \%$ and the fellow eye (control eye) received artificial tears. Each topical medication was applied three times with 10-min intervals. ChT measurements were performed at baseline and $40 \mathrm{~min}$ after the last drops of the topical medications by enhanced depth imaging (EDI) optical coherence tomography (OCT).

Results In the cyclopentolate group, subfoveal ChT significantly increased in the study eyes $(P=0.013)$, whereas it did not significantly change in the control eyes $(P=0.417)$. On the other hand, in the tropicamide group, no significant subfoveal ChT changes were observed in either the study eyes $(P=0.715)$ or the control eyes $(P=0.344)$.

Conclusions The current study demonstrated that cyclopentolate caused significant choroidal thickening, whereas tropicamide had no significant effect on ChT in healthy

Eye (2016) 30, 925-928; doi:10.1038/eye.2016.61; published online 8 April 2016

\section{Introduction}

The choroid is the highly vascular layer of the eye placed between retina and sclera. It has several functions including procurement of oxygen and nutrients for the outer retina, adjustment of retinal position, regulation of retinal temperature, protection of the photoreceptors, and secretion of some growth factors. ${ }^{1,2}$ The introduction of enhanced depth imaging (EDI) optical coherence tomography (OCT) technology by Spaide et $a l^{3}$ has provided accurate measurement of choroidal thickness (ChT) for investigating the in vivo anatomy of normal choroid and the choroidrelated ocular pathologies.

Previous studies by EDI OCT have shown that ChT is influenced by numerous ocular disorders including central serous chorioretinopathy, ocular tumors, age-related macular degeneration, diabetic retinopathy, and inflammatory eye diseases. ${ }^{4-8}$ In addition, it is known that ChT is affected by various factors such as blood pressure, age, gender, time of the day, refractive status, axial length, and forced hyperopic or myopic defocus. ${ }^{2,9-11}$ Further, a recent experimental study showed that anti-muscarinic agents could affect ChT. ${ }^{12}$ Therefore, in the present study, we aimed to investigate the effects of tropicamide and cyclopentolate, which are two anti-muscarinic agents commonly used in the ophthalmologic practice, on subfoveal ChT measured by EDI OCT in healthy adults.
\end{abstract} adults. As a result, mydriasis by cyclopentolate may complicate ChT measurements by EDI OCT. Use of tropicamide may provide more reliable results for evaluation of $\mathrm{ChT}$ in ocular pathologies.
Materials and methods

A total of 74 healthy adult subjects recruited from ophthalmology outpatient clinic were enrolled in the study. Subjects were randomly
Department of

Ophthalmology, Recep Tayyip Erdoğan University Medical School, Rize, Turkey

Correspondence:

$\checkmark$ Öner, Department of Ophthalmology, Recep Tayyip Erdoğan University Medical School, Rize 53100, Merkez, Turkey Tel: +90 4642130491 ; Fax: +90 4642170364 . E-mail: veysioner@gmail. com

Received: 9 January 2016 Accepted in revised form: 4 February 2016 Published online: 8 April 2016 
divided into two groups: (1) cyclopentolate group ( $n=37)$ in which the right eye (study eye) of each subject received topical cyclopentolate $1 \%$, and the fellow eye (control eye) received artificial tears and (2) tropicamide group $(n=37)$ in which the right eye (study eye) of each subject received topical tropicamide $1 \%$ and the fellow eye (control eye) received artificial tears. Each topical medication was applied three times with 10-min intervals in both groups. Exclusion criteria were smoking, alcohol use, any ocular disorder reducing visual acuity, any ocular or systemic disorder that might affect ChT measurement (ie, corneal scar, congenital cataract, glaucoma, strabismus, vitreoretinal disorders, diabetes mellitus), myopia or hyperopia $\geq 6$ diopters (D), astigmatism $\geq 2 \mathrm{D}$, a history of ocular trauma or surgery, age $<16$ years, and poor image quality. All subjects were informed about the study and they admitted to take part. The study was in accordance with the rules of the Declaration of Helsinki and was approved by the local ethics committee.

Before the application of the study medications (at baseline), subjects underwent an ophthalmologic examination including assessment of refractive status and visual acuity, slit-lamp examination, measurement of intraocular pressure, fundoscopy, and quantification of subfoveal ChT. The ChT of each eye was remeasured $40 \mathrm{~min}$ after the last drop of the topical medication.

ChT was assessed by the HD 5 Line Raster scan protocol of spectral domain EDI OCT (Cirrus HD, Carl Zeiss Meditec Ophthalmic Systems Inc., Dublin, CA, USA). The assessment was performed manually as the distance between the retinal pigment epithelium and the chorioscleral border, by two independent blinded investigators, between 1030 hours and 1200 hours. As the assessments of the two investigators were statistically similar, the results of one investigator were used in the study.

\section{Statistical analysis}

Statistical analysis was performed by using the Statistical Package for the Social Sciences (SPSS Inc., Chicago, IL, USA) version 16. Kolmogorov-Smirnov test was used for evaluation of the distributions of the variables. Paired t-test was used to compare the ChT values. The statistical significance was set at $P<0.05$.

\section{Results}

The mean age was $43.8 \pm 16.5$ years (from 16 to 64 years) in the cyclopentolate group and $41.4 \pm 14.3$ years (from 16 to 66 years) in the tropicamide group. There were 20 females and 17 males in the cyclopentolate group and 19 females and 18 males in the tropicamide group. All subjects had visual acuities of at least 1.0 (Snellen notations) in both eyes. In the cyclopentolate group, the mean spherical equivalent was $+0.68 \pm 1.4$ (from -2.50 to +4.25 ) in the study eyes and $+0.62 \pm 1.3$ (from -2.50 to +4.00 ) in the control eyes. In the tropicamide group, the mean spherical equivalent was $+0.36 \pm 0.8$ (from -1.00 to +2.75 ) in the study eyes and $+0.29 \pm 0.0$ (from -1.25 to +2.50 ) in the control eyes.

The subfoveal ChT values of the subjects are given in Table 1. In the cyclopentolate group, subfoveal ChT significantly increased after the use of cyclopentolate in the study eyes $(P=0.013)$, whereas it did not significantly change after the use of artificial tears in the control eyes $(P=0.417)$. On the other hand, in the tropicamide group, no significant subfoveal ChT changes were observed in either the study eyes after the use tropicamide $(P=0.715)$ or the control eyes after artificial tears administration $(P=0.344)$.

\section{Discussion}

Cyclopentolate $1 \%$ and tropicamide $1 \%$ are antimuscarinic (parasympatholytic) drugs that are widely used in ophthalmologic practice to create cycloplegia before the evaluation of refraction, to dilate pupil before fundoscopy, surgery, or fundus fluorescein angiography, and to prevent or break posterior synechia in eyes with uveitis. The present study showed that although tropicamide had no significant effect on ChT,

Table 1 The subfoveal choroidal thickness values of the subjects (mean \pm s.d.)

\begin{tabular}{lrrr}
\hline Group & Preapplication & Postapplication & P-value \\
\hline Cyclopentolate group $(n=37)$ & & & \\
$\quad$ Study eye & $328.16 \pm 68.7(194$ to 570$)$ & $349.7 \pm 86.6(221$ to 603$)$ & $\mathbf{0 . 0 1 3}$ \\
$\quad$ Control (fellow) eye & $322.1 \pm 56.2(224$ to 505$)$ & & 0.417 \\
& & $311.2 \pm 73.1(169$ to 545$)$ & \\
Tropicamide group $(n=37)$ & $308.5 \pm 63.6(202$ to 513$)$ & $316.2 \pm 78.2(170$ to 524$)$ & 0.715 \\
$\quad$ Study eye & $322.7 \pm 70.1(170$ to 510$)$ & & 0.344 \\
$\quad$ Control (fellow) eye & & & \\
\hline
\end{tabular}

Significant $P$-values are given in bold. 
cyclopentolate caused significant rapid choroidal thickening in healthy adults.

Although the choroid is structurally constituted by the branches of ciliary arteries, ${ }^{13}$ it also includes non-vascular smooth muscle cells, and a network of spindle-shaped or star-shaped cells. ${ }^{1,14}$ These cells are considered to have a role in changing the $\mathrm{ChT}$ in proportion to the effects of local mediators and sympathetic and parasympathetic systems. ${ }^{1,14}$ Nickla and Schrodl ${ }^{15}$ demonstrated that double parasympathectomy caused choroidal thickening in chick eyes. This result indicates that parasympathetic innervation and non-vascular smooth muscle may have a role in the choroidal thickening response. In addition, in a study by Nickla $e t a l,{ }^{12}$ in intact chick eyes, intravitreal injections of anti-muscrinic agents (atropine, pirenzepine, and oxyphenonium) inhibited ocular elongation in response to negative lenses, and also resulted in a rapid, transient choroidal thickening. They also revealed that intravitreal injections of muscarinic agonists caused choroidal thinning in eyecups without retinas and this was a sign of a muscarinic manner of action on either the retinal pigment epithelium or the choroid, both tissues that have been shown to contain muscarinic cholinergic receptors. ${ }^{16}$ In accordance with these findings, in our study, cyclopentolate caused a significant rapid choroidal thickening in healthy human eyes in which choroidal non-vascular smooth muscle also has parasympathetic innervation. ${ }^{15}$

In accordance with our results, Sander et $a l^{17}$ found that the application of homatropine $2 \%$ increased subfoveal $\mathrm{ChT}$ significantly. Acetylcholine is a potent vasodilator, which is secreted from parasympathetic cholinergic nerves. However, experimental studies have shown that anticholinergic agents such as atropine could also cause vasodilation in ocular vessels. ${ }^{17,18}$ This inverse effect of the anticholinergic agents may be explained by their effect of enhancing the secretion of neural nitric oxide via prejunctional muscarinic receptors; and as a result, the choroidal thickening associated with anticholinergic agents may result from the presynaptic inhibition of muscarinic receptors, increased neural nitric oxide levels, and consequent relaxation of smooth muscles. ${ }^{17,18}$

We found that tropicamide had no significant effect on ChT. Compared with cyclopentolate, tropicamide is a cycloplegic drug with lower potency, rapid effect and shorter duration of action. These features might make the effect of tropicamide to be insufficient for achieving an alteration in ChT, in our study. In accordance with our results, Mwanza et al ${ }^{19}$ revealed that no significant change occurred in ChT after the application of topical tropicamide in both normal and glaucomatous eyes. In addition, Kim et $a l^{20}$ evaluated the effect of Mydrin-P, which is composed of of tropicamide and phenylephrine, and showed that it had no significant effect on subfoveal ChT measured by EDI OCT.

Posterior uveitis and panuveitis such as Behçet's disease and Vogt-Koyanagi-Harada syndrome may involve the choroid as well as adjacent structures such as the retina, vitreous, and blood vessels. Previous studies, by using EDI OCT, have shown choroidal thickening in acute phases of both Behçet's disease ${ }^{21}$ and Vogt-Koyanagi-Harada syndrome. ${ }^{22,23}$ It is known that mydriatic agents including cyclopentolate can be used not only for the pupil dilation before the evaluation of ChT by EDI OCT but also for the treatment ${ }^{24}$ of these aforementioned diseases. Our results showed that the choroidal thickening effect of cyclopentolate should be kept in mind when evaluating ChT in patients with posterior or panuveitis.

The limitation of the current study was the manual measurement of ChT. All studies investigating ChT by EDI OCT use manual measurements owing to lack of commercially available automated software. Although it has been demonstrated that the manual assessments have perfect reliability, there may be significant variations among investigators in some parameters. ${ }^{25}$ Therefore, in our study, to check the reliability, the evaluations were carried out by two independent investigators and were compared.

In conclusion, our study demonstrated that cyclopentolate caused significant choroidal thickening, whereas tropicamide had no significant effect on ChT in healthy adults. As a result, mydriasis by cyclopentolate may complicate ChT evaluation by EDI OCT. Use of tropicamide may provide more reliable results for evaluation of $\mathrm{ChT}$ in ocular pathologies.

\section{Summary}

What was known before

- Choroidal thickness is affected by various factors such as blood pressure, age, gender, time of the day, refractive status, axial length, and forced hyperopic or myopic defocus.

\section{What this study adds}

- Cyclopentolate causes a significant rapid choroidal thickening whereas tropicamide has no significant effect on choroidal thickness in healthy adults.

\section{Conflict of interest}

The authors declare no conflict of interest.

\section{References}

1 Nickla DL, Wallman J. The multifunctional choroid. Prog Retin Eye Res 2010; 29: 144-168.

2 Wallman J, Wildsoet C, Xu A. Moving the retina: choroidal modulation of refractive state. Vision Res 2006; 46: 267-283. 
3 Spaide RF, Koizumi H, Pozzoni MC. Enhanced depth imaging spectral-domain optical coherence tomography. Am J Ophthalmol 2008; 146: 496-500.

4 Yang L, Jonas JB, Wei W. Optical coherence tomographyassisted enhanced depth imaging of central serous chorioretinopathy. Invest Ophthalmol Vis Sci 2013; 54: 4659-4665.

5 Shields CL, Manalac J, Das C, Saktanasate J, Shields JA. Review of spectral domain enhanced depth imaging optical coherence tomography of tumors of the choroid. Indian J Ophthalmol 2015; 63: 117-121.

6 Capuano V, Souied EH, Miere A, Jung C, Costanzo E, Querques G. Choroidal maps in non-exudative age-related macular degeneration. Br J Ophthalmol; epub ahead of print 7 September 2015; doi:10.1136/bjophthalmol-2015-307169.

7 Kase S, Endo H, Yokoi M, Kotani M, Katsuta S, Takahashi M et al. Choroidal thickness in diabetic retinopathy in relation to long-term systemic treatments for diabetes mellitus. Eur J Ophthalmol 2015; 26: 158-162.

8 Coskun E, Gurler B, Pehlivan Y, Kisacik B, Okumus S, Yayuspayı $\mathrm{R}$ et al. Enhanced depth imaging optical coherence tomography findings in Behçet disease. Ocul Immunol Inflamm 2013; 21: 440-445.

9 Akay F, Gundogan FC, Yolcu U, Toyran S, Uzun S. Choroidal thickness in systemic arterial hypertension. Eur J Ophthalmol; epub ahead of print 8 September 2015; doi:10.5301/ejo.5000675.

10 Herrera L, Perez-Navarro I, Sanchez-Cano A, Perez-Garcia D, Remon L, Almenara C et al. Choroidal thickness and volume in a healthy pediatric population and its relationship with age, axial length, ametropia, and sex. Retina 2015; 35: 2574-2583.

11 Chakraborty R, Read SA, Collins MJ. Diurnal variations in axial length, choroidal thickness, intraocular pressure, and ocular biometrics. Invest Ophthalmol Vis Sci 2011; 52: 5121-5129.

12 Nickla DL, Zhu X, Wallman J. Effects of muscarinic agents on chick choroids in intact eyes and eyecups: evidence for a muscarinic mechanism in choroidal thinning. Ophthalmic Physiol Opt 2013; 33: 245-256.

13 Bhutto IA, Amemiya T. Microvascular architecture of the rat choroid: corrosion cast study. Anat Rec 2001; 264: 63-71.

14 Lütjen-Drecoll E. Choroidal innervation in primate eyes. Exp Eye Res 2006; 82: 357-361.
15 Nickla DL, Schroedl F. Parasympathetic influences on emmetropization in chicks: evidence for different mechanisms in form deprivation vs negative lens-induced myopia. Exp Eye Res 2012; 102: 93-103.

16 Rada JA, McFarland AL, Cornuet PK, Hassell JR. Proteoglycan synthesis by scleral chondrocytes is modulated by a vision dependent mechanism. Curr Eye Res 1992; 11: 767-782.

17 Sander BP, Collins MJ, Read SA. The effect of topical adrenergic and anticholinergic agents on the choroidal thickness of young healthy adults. Exp Eye Res 2014; 128: 181-189.

18 Ayajiki K, Tanaka T, Okamura T, Toda N. Evidence for nitroxidergic innervation in monkey ophthalmic arteries in vivo and in vitro. Am J Physiol Heart Circ Physiol 2000; 279: H2006-H2012.

19 Mwanza JC, Sayyad FE, Banitt MR, Budenz DL. Effect of pupil dilation on macular choroidal thickness measured with spectral domain optical coherence tomography in normal and glaucomatous eyes. Int Ophthalmol 2013; 33: 335-341.

20 Kim M, Kwon HJ, Lee SC. Influence of mydriatics on choroidal thickness measurement using enhanced depth imaging-OCT. Optom Vis Sci 2012; 89: 1150-1155.

21 Kim M, Kim H, Kwon HJ, Kim SS, Koh HJ, Lee SC. Choroidal thickness in Behcet's uveitis: an enhanced depth imaging-optical coherence tomography and its association with angiographic changes. Invest Ophthalmol Vis Sci 2013; 54: 6033-6039.

22 Fong AH, Li KK, Wong D. Choroidal evaluation using enhanced depth imaging spectral-domain optical coherence tomography in Vogt-Koyanagi-Harada disease. Retina 2011; 31: 502-509.

23 Maruko I, Iida T, Sugano Y, Oyamada H, Sekiryu T, Fujiwara $\mathrm{T}$ et al. Subfoveal choroidal thickness after treatment of Vogt-Koyanagi-Harada disease. Retina 2011; 31: 510-517.

24 Chan RV, Lee TC, Chaganti RK, Cestari DM, Kim MT, Lee S. Macular star associated with Behçet disease. Retina 2006; 26: 468-470.

25 Sanchez-Cano A, Orduna E, Segura F, Lopez C, Cuenca N, Abecia $\mathrm{E}$ et al. Choroidal thickness and volume in healthy young white adults and the relationships between them and axial length, ammetropy and sex. Am J Ophthalmol 2014; 158: 574-583. 